\title{
Inversion of the Coupling Absorption at the Two-Photon Resonance in a Coupling-Probe-Spectroscopy Experiment
}

\author{
K. Dahl, L. Spani Molella, R.-H. Rinkleff, K. Danzmann \\ Albert Einstein Institute, Max Planck Institute for Gravitational Physics \\ and Institut für Gravitationsphysik, Gottfried Wilhelm Leibniz Universität Hannover \\ Callinstraße 38, D-30167 Hannover, Germany. \\ Tel: +49 5117625843 ; Fax: +49 511762 2784; E-mail: rolf-hermann.rinkleff@aei.mpg.de
}

After the discovery of electromagnetically induced absorption (EIA), both theoretical and experimental research was performed to explain not only the probe laser absorption and dispersion spectra, but also those of the coupling laser. The investigations of EIA in degenerate two-level systems have often been realized using two linearly polarized lasers of orthogonal polarization [1]. However, the simplest configuration leading to EIA is the so-called $\mathrm{N}$-system [2] which basically requires the simultaneous adoption of a circularly polarized coupling laser and a linearly polarized probe (here called $\sigma-\pi$ ). To our knowledge, no experimental measurements of the coupling laser exist in the configurations $\left(\sigma^{+}-\sigma^{-}, \pi-\sigma^{-}\right.$or $\left.\sigma^{+}-\pi\right)$.

In our contribution we study the coupling and probe absorption spectra as a function of the probe detuning also for the case in which the probe intensity was high enough to affect the pump absorption. We present a complete experimental survey of the coupling and probe absorption spectra for all polarization combinations. The measurements were performed within the $\mathrm{D}_{2}$ line of cesium, on the hyperfine transition $6 \mathrm{~s}^{2} \mathrm{~S}_{1 / 2}, \mathrm{~F}=4$ to $6 \mathrm{p}{ }^{2} \mathrm{P}_{3 / 2}, \mathrm{~F}$ $=5$. To minimize the Doppler broadening of the signals, an atomic beam of cesium atoms propagating perpendicularly to the direction of propagation of the lasers was adopted, instead of using a conventional cell. The setup was a simplified form of the one described in $[3,4]$.

As for the case of double-linear orthogonal polarizations, the absorption spectra of the coupling laser showed in the region around the two-photon resonance a broad transparency window below the one-photon absorption mainly accompanied by a narrow feature at the two-photon resonance. In case of double linear polarization we named this behavior "absorption within transparency". Similar spectra were measured both in the case of circularly polarized lasers of right-hand and left-hand polarization $\left(\sigma^{+}-\sigma^{-}\right)$and in the two linear-circular cases $\left(\pi-\sigma^{-}\right.$and $\left.\sigma^{+}-\pi\right)$. Surprisingly, the narrow feature at the two-photon resonance in the $\sigma^{+}-\sigma^{-}$case switches from an absorption peak to a transparency dip once the probe intensity gets greater than that of the coupling laser, i.e. once the probe assumes a pumping role in the system. Thus in the case of the coupling spectra, one measures a switch from "absorption within transparency" into "transparency within transparency".. Some of the results obtained agree well with the theoretical predictions given in [2], thus confirming the key role played by spontaneous transfer of coherence in the generation of the spectra.

All spectra were measured for various intensities of both lasers. Depending on the measured intensity ranges it was possible to evidence a change in the width of the broad transparency signals accompanied by a variation in the intensity of the central narrow-line feature.

The work was supported by the grant no. SFB407 of the Deutsche Forschungsgemeinschaft.

[1] L. Spani Molella, R.-H. Rinkleff, and K. Danzmann, "Giant Kerr effect in closed degenerate two-level transitions", Appl. Phys. B, 90, 273 (2008)

[2] C. Goren, A. D. Wilson-Gordon, M. Rosenbluh, and H. Friedmann, “Atomic four-level N systems", Phys. Rev. A 69, 053818 1-10 (2004)

[3] L. Spani Molella, R.-H. Rinkleff, and K. Danzmann, "Role of the coupling laser in electromagnetically induced transparency", Phys Rev. A 72, 041802(R) 1-4 (2005)

[4] L. Spani Molella, R.-H. Rinkleff, and K. Danzmann, "Spectral measurement of the caesium $\mathrm{D}_{2}$ line with a tunable heterodyne interferometer", Spectrochim. Acta A 63, 987-993 (2006) 